Research Article

\title{
The Method of Quantitative Trend Diagnosis of Rolling Bearing Fault Based on Protrugram and Lempel-Ziv
}

\author{
Jianxi Du, ${ }^{1}$ Lingli Cui $\mathbb{D}^{1},{ }^{1}$ Jianyu Zhang $\mathbb{D}^{1},{ }^{1}$ Jin $\mathrm{Li}^{2},{ }^{2}$ and Jinfeng Huang ${ }^{2}$ \\ ${ }^{1}$ Key Laboratory of Advanced Manufacturing Technology, Beijing University of Technology, Chao Yang District, \\ Beijing 100124, China \\ ${ }^{2}$ Beijing Engineering Research Center of Precision Measurement Technology and Instruments, \\ Beijing University of Technology, Beijing 100124, China \\ Correspondence should be addressed to Jianyu Zhang; zhjy_1999@bjut.edu.cn
}

Received 4 May 2018; Revised 4 July 2018; Accepted 28 August 2018; Published 1 November 2018

Academic Editor: Adam Glowacz

Copyright (c) 2018 Jianxi Du et al. This is an open access article distributed under the Creative Commons Attribution License, which permits unrestricted use, distribution, and reproduction in any medium, provided the original work is properly cited.

\begin{abstract}
This paper proposes a new method to realize the quantitative trend diagnosis of bearings based on Protrugram and Lempel-Ziv. Firstly, the fault features of original fault signals of bearing inner and outer race with different severity are extracted using Protrugram algorithm, and the optimal analysis frequency band is selected which reflects the fault characteristic. Then, the Lempel-Ziv complexity of the frequency band is calculated. Finally, the relationship between Lempel-Ziv complexity and fault size is obtained. Analysis results show that the severity of fault is proportional to the Lempel-Ziv complexity index value under different fault types. The Lempel-Ziv complexity showed different trend rules, respectively, in the inner and outer race, which realized the quantitative trend diagnosis of bearing faults.
\end{abstract}

\section{Introduction}

Rolling bearings are important components in rotating machinery [1]. Regrettably, they are also prone to premature failure due to a copious amount of possible influence factors $[2,3]$. Consequently, the detection and diagnosis of rolling bearing faults are of great significance [4]. At present, the research on bearing fault diagnosis based on vibration signals mainly focuses on two aspects: the research on feature extraction method of bearing vibration signal and study on vibration mechanism of bearing fault [5]. Most of these methods are qualitative analysis of bearing fault, but quantitative diagnosis of bearing fault is relatively few. The development of modern equipment is becoming more complicated and precise, so the quantitative analysis of equipment failures is particularly important.

In recent years, the quantitative analysis of bearing fault and damage severity has been widely concerned by scholars both at home and abroad. For example, Jiang et al. [6] obtained the relationship between the complexity and fault severity of bearing signal and applied the improved morphologic filtering method to the noise reduction and the extraction of the complexity index of bearing fault signals. Zhang et al. [7] used the permutation entropy index based on EEMD (ensemble empirical mode decomposition) to classify the bearing failure types and damage severity. Cui et al. [8] proposed a quantified matching pursuit algorithm based on new dictionary model for bearing fault diagnosis, which can effectively measure the size of the spalling fault of bearings and realize the quantitative diagnosis of rolling bearing faults. A rolling element bearing in operation can be considered as a nonlinear dynamical system, and the nonlinearity of a system is typically described using the complexity index of the system. At present, many scholars applied Lempel-Ziv complexity to calculate the complexity of finite-length time series. Lempel-Ziv index is a new research direction for quantitative diagnosis of bearing failure. Gao et al. [9] measured the complexity of gear vibration signals using the Lempel-Ziv, and the results show that the complexities of the normal gear and the fatigue spalling gear are significantly different, and the complexity measure may be the characteristic parameter of fault diagnosis, which 
deserves further study. Hong and Liang [10] proposed a new version of Lempel-Ziv complexity based on continuous wavelet transform to measure the bearing fault severity, and the result shows that, for all rotational speeds, the complexity was proportional for outer race fault and inversely proportional for inner race fault to failure size. Dou and Zhao [11] proposed an evaluation method based on empirical mode decomposition (EMD) and Lempel-Ziv index and obtained the range of Lempel-Ziv value to evaluate different faults of inner and outer race damage. Zhang and Chen [12] proposed a method based on LMD and Lempel-Ziv to identify the damage severity of rolling bearings, for different rotation speeds and different inner or outer race defects severity. The above analysis shows that mechanical fault signal contains information about the severity of fault. Therefore, the quantitative analysis of the bearing fault severity is necessary. Proper maintenance decision can be made only when fault severity is accurately assessed.

However, in practical engineering, the signal features are very weak and vulnerable to the influence of strong background noise $[13,14]$, which is a typical nonstationary signal [15]. At this point, not all frequency bands in the signal have obvious fault characteristics. Under strong background noise, the frequency band selection is often subject to interference resulting in poor accuracy of center frequency and filter bandwidth. Barszcz and Jabłoński [16] pointed out that spectral kurtosis was not applicable to non-Gauss and strong noise; in order to solve this problem, the Protrugram algorithm is proposed, which selects the optimal frequency band by calculating the kurtosis of envelope spectrum amplitude and verifies the validity of the method to extract fault features in the case of non-Gaussian and strong noise. Therefore, we apply the Protrugram algorithm to select the optimal center frequency and bandwidth of the original signal with different fault sizes. The Protrugram is used to select the optimal frequency band, which is designed to select the optimal center frequency and bandwidth [17].

In view of the above, the Protrugram and Lempel-Ziv are applied to the quantitative trend diagnosis of rolling bearing failure. Firstly, the fault features of the signals are extracted, and the frequency band is selected. Then, the Lempel-Ziv index is used to measure the signal complexity. Finally, the relationship between Lempel-Ziv complexity and fault size is obtained. The complexity of bearing vibration signal is quantified to evaluate the state of bearing and realizes the quantitative trend diagnosis of bearing fault.

\section{Fault Mechanism of Rolling Bearings}

2.1. Simulation Signals for Different Fault Sizes of Rolling Bearings. The rolling bearings are mainly composed of four parts: inner race, outer race, rolling element, and cage. In the process of rolling bearing installation and operation, the outer race is connected with the bearing seat or box, and the inner race is connected with the shaft neck of the drive shaft and turns with the shaft. When the shaft runs at a certain speed and a certain load, it will stimulate the vibration system composed of bearing, bearing seat, and box [18].
In the case of the small area of the local damage of the rolling bearing, it is assumed that the impact structure produced by the fault is an ideal impulse similar to the traditional impact dictionary model. However, when the area of local damage increases, the pulse caused by the fault cannot be in an ideal state, but it has a certain width. The rising edge of the pulse can be considered as the state when the rolling element is just in contact with the fault edge, and the falling edge of the pulse can be considered as the state when the rolling element leaves the other edge of the fault, as shown in Figure 1. When the fault has a certain width, the rolling element will have two impacts on the edge of the fault through the fault location.

In order to get the precise impact model, a simulation signal which can reflect the size of the fault is established. Firstly, we need to calculate the linear speed of the rolling elements during motion and the pulse width caused by different faults.

The linear speed of rolling element: $s=\pi d f_{\mathrm{r}}$; pulse width: $p_{x}=\left(d_{x} / s\right)$.

The pulse generated by the defect can be expressed as [19]

$$
\begin{gathered}
x(t)= \begin{cases}1, & u<t<u+p_{x}, \\
0,\end{cases} \\
\varnothing_{\mathrm{imp}}(p, u, f)= \begin{cases}K_{\mathrm{imp}} e^{-p(t-u)} \sin 2 \pi f t, & t \geq u, \\
0, & t<u,\end{cases}
\end{gathered}
$$

where $u$ is the initial moment when the impact response occurs (s); $f$ is the damping natural frequency of the system $(\mathrm{Hz}) ; p$ is the damping attenuation characteristics of impact response; and $K_{\text {imp }}$ is the normalization constant.

According to the above theory, the simulation signal of bearing fault is processed. The pulse sequence produced by the defect of the bearing vibration signal is

$$
x(t)=\left\{\begin{array}{l}
y, n\left(\frac{1}{f_{\mathrm{d}}}\right)<t<n\left(\frac{1}{f_{\mathrm{d}}}\right)+p_{x}, \\
0,
\end{array}\right.
$$

where $p_{x}$ is the pulse width $(\mathrm{mm}) ; y$ is the amplitude $\left(\mathrm{mm} / \mathrm{s}^{2}\right)$; and $f_{\mathrm{d}}$ is the fault characteristic frequency $(\mathrm{Hz})$.

Therefore, the vibration signal model of the outer race pitting failure can be simplified as

$$
\phi_{\text {imp }}\left(p, u, f, d_{x}, d, f_{\mathrm{r}}\right)=\operatorname{conv}\left(x_{\mathrm{f}}(t), \phi_{\text {imp }}(p, u, f)\right) .
$$

Based on the model, the simulation signal with pitting fault in outer race is established, in which the motor speed is $R=1496 \mathrm{r} / \mathrm{min}$, the bearing outside diameter $D=80 \mathrm{~mm}$, the bearing inner diameter $r d=35 \mathrm{~mm}$, the number of rolling elements $Z=8$, and the contact angle $\alpha=0$. The sampling frequency $f_{\mathrm{s}}$ is $25.6 \mathrm{kHz}$ and sampling number $N$ is 8192. According to the above parameters, the fault characteristic frequency of bearing outer race is calculated to be $76.7282 \mathrm{~Hz}$, and the characteristic frequency of inner race fault is $122.738 \mathrm{~Hz}$. According to the above theory, the 


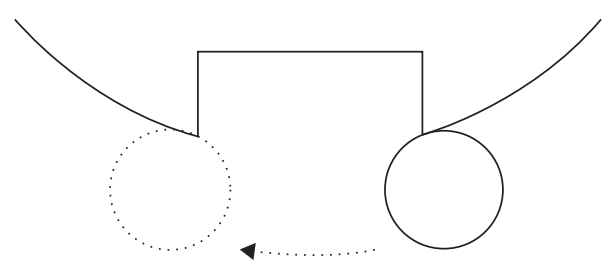

Figure 1: Physical model diagram.

simulation signals of different fault sizes of rolling bearings are constructed, the size of the fault is $0.5 \mathrm{~mm}, 2 \mathrm{~mm}$, $3.5 \mathrm{~mm}$, and $5 \mathrm{~mm}$, and the signals are added with $2 \mathrm{~dB}$ noise. The inner race fault signals are shown in Figure 2, and the outer race fault signals are shown in Figure 3.

2.2. The Fault Characteristic of the Bearing. Faults in rolling bearings are usually caused by local defects in the outer race, inner race, and roller. A series of shock vibrations are generated when the running rolling element passes through the defect surface. The characteristic frequencies of a bearing are calculated based on the bearing geometry and the rotor frequency $f_{\mathrm{r}}$. By comparing the fault characteristic frequencies in the spectra with calculated characteristic frequencies of bearing, the cause of the defect can be identified.

Outer race defects are revealed at the outer race pass frequency $\left(f_{0}\right)$ :

$$
f_{\mathrm{o}}=\frac{Z}{2}\left(1-\frac{d}{D} \cos \alpha\right) f_{\mathrm{r}}
$$

Inner race defects are revealed at the inner race pass frequency $\left(f_{\mathrm{i}}\right)$ :

$$
f_{\mathrm{i}}=\frac{Z}{2}\left(1+\frac{d}{D} \cos \alpha\right) f_{\mathrm{r}},
$$

where $d$ is the diameter of rollers, $D$ is the pitch diameter, $Z$ is the number of rollers, $\alpha$ is the contact angle of the rollers, and $f_{\mathrm{r}}$ is the rotation frequency.

\section{The Proposed Method of Quantitative Trend Diagnosis}

3.1. The Lempel-Ziv Algorithm. Lempel-Ziv was first proposed by mathematicians Lempel and Ziv [10]. Lempel-Ziv complexity is a powerful tool for measuring the complexity of finite time series [20]. The basic theory of Lempel-Ziv is with the increase of the complexity of the sequence, the periodic components in the sequence become less, and the sequence becomes more irregular, at the same time, and it approaches the random state. The sequence contains more frequency components, indicating that the system is more complex. When the complexity of the sequence becomes smaller, the periodic components of the sequence become clearer and tend to become more periodic. The sequence contains fewer frequency components, indicating that the complexity of the system will be lower.

For a signal $S(i) \quad(i=1,2, \ldots, N)[21]$, firstly, convert it into a binary sequence, and set $a=$ mean $(S(i)) \varepsilon$, if $S(i) \geq 0$, then $S(i)=0$, else $S(i)$. So transform $S(i)$ into binary sequence $S_{N}=\left\{s_{1}, s_{2}, s_{3}, \ldots, s_{N}\right\}$, and Lempel-Ziv complexity value of sequence $S_{N}$ can be calculated by cycling $N$ times with $C_{N}(r) \quad(r \leq N)$ :

(1) Initialization, $S_{v, 0}\{\}, Q_{0}=\{\}, C_{N}(0)=0[11], r=0$, makes $Q_{r}=\left\{Q_{r-1} S_{r}\right\}$, since $Q_{0}$ does not belong to $S_{v, r-1}$, then $C_{N}(r)=C_{N}(r-1)+1, Q_{r}=\{\}$, $r=r+1$

(2) Make $Q_{r}=\left\{Q_{r-1} S_{r}\right\}$, judging weather $Q_{r}$ belongs to $S_{v, r-1}=\left\{S_{v, r-2} s_{r-1}\right\}, \quad$ if so, $C_{N}(r)=C_{N}(r-1)$, $r=r+1$, repeat step (2);

(3) If not, $\mathrm{C}_{N}(r)=\mathrm{C}_{N}(r-1)+1, \mathrm{Q}_{r}=\{\}, r=r+1$, repeat step (2).

Take $S_{N}=\left\{\begin{array}{llllllll}1 & 0 & 0 & 1 & 0 & 1 & 1 & 0\end{array}\right\}$ as an example, $N=8$, initial $r=0, S_{v, 0}=\{\}, Q_{0}=\{\}, C_{N}(0)=0$.

$r=1, S_{v, 0}=\{\}, Q_{1}=\left\{Q_{0} s_{1}\right\}=\{1\}, Q_{1} \notin S_{v, 0}, C_{N}(1)=$ $C_{N}(0)+1=1, Q_{1}=\{\}$

$r=2, S_{v, 1}=\left\{S_{v, 0} s_{1}\right\}=\{1\}, Q_{2}=\left\{Q_{1} s_{2}\right\}=\{10\}, Q_{2} \notin$

$S_{v, 1}, C_{N}(2)=C_{N}(1)+1=2, Q_{2}=\{\}$

$r=3, S_{v, 2}=\left\{S_{v, 1} s_{2}\right\}=\{10\}, Q_{3}=\left\{Q_{2} s_{3}\right\}=\{0\}, Q_{3} \in S_{v, 2}$,

$C_{N}(3)=C_{N}(2)=2$

$r=4, S_{v, 3}=\left\{S_{v, 2} s_{3}\right\}=\left\{\begin{array}{lll}1 & 0 & 0\end{array}\right\}, Q_{4}=\left\{Q_{3} s_{4}\right\}=\left\{\begin{array}{lll}0 & 1\end{array}\right\}$,

$Q_{4} \notin S_{v, 3}, C_{N}(4)=C_{N}(3)+1=3, Q_{4}=\{\}$

$r=5, S_{v, 4}=\left\{S_{v, 3} s_{4}\right\}=\left\{\begin{array}{llll}1 & 0 & 0 & 1\end{array}\right\}, Q_{5}=\left\{Q_{4} s_{5}\right\}=\left\{\begin{array}{ll}0\end{array}, Q_{5} \in\right.$

$S_{v, 4}, C_{N}(5)=C_{N}(4)=3$

$r=6, S_{v, 5}=\left\{S_{v, 4} s_{5}\right\}=\left\{\begin{array}{lllll}1 & 0 & 0 & 1 & 0\end{array}\right\}, Q_{6}=\left\{Q_{5} s_{6}\right\}=\left\{\begin{array}{lll}0 & 1\end{array}\right\}$,

$Q_{6} \in S_{v, 5}, C_{N}(6)=C_{N}(5)=3$

$r=7, S_{v, 6}=\left\{S_{v, 5} s_{6}\right\}=\left\{\begin{array}{llllll}1 & 0 & 0 & 1 & 0 & 1\end{array}\right\}, Q_{7}=\left\{Q_{6} s_{7}\right\}=\left\{\begin{array}{lll}0 & 1\end{array}\right.$

$1\}, Q_{7} \notin S_{v, 6}, C_{N}(7)=C_{N}(6)+1=4, Q_{7}=\{\}$

$r=8, S_{v, 7}=\left\{S_{v, 6} s_{7}\right\}=\left\{\begin{array}{lllllll}1 & 0 & 0 & 1 & 0 & 1 & 1\end{array}\right\}, Q_{8}=\left\{Q_{7} s_{8}\right\}=\{0\}$,

$Q_{8} \in S_{v, 7}, C_{N}(8)=C_{N}(7)=4$.

Through the above cycle, the Lempel-Ziv complexity value of $S_{N}$ is 4 .

The complexity value related with the length $N$ of $S_{N}$, $C_{N}(N)$ is affected by length $N$ of $S_{N}$. In order to make Lempel-Ziv complexity index relatively independent, Lempel and Ziv proposed the following normalized formula [11]:

$$
\begin{aligned}
0 \leq C_{n N} & =\frac{C_{N}(N)}{\lim _{N \longrightarrow \infty} C_{N}(N)}=\frac{C_{N}(N)}{\lim _{N \longrightarrow \infty}\left(N /(1-\beta) \log _{k} N\right)} \\
& \approx \frac{C_{N}(N) \times \log _{k} N}{N}
\end{aligned}
$$

where $k$ is the number of elements in $S_{N}$ (for binary sequence $S_{N}, k=2$ ). Hong et al. [10] gave the empirical value 


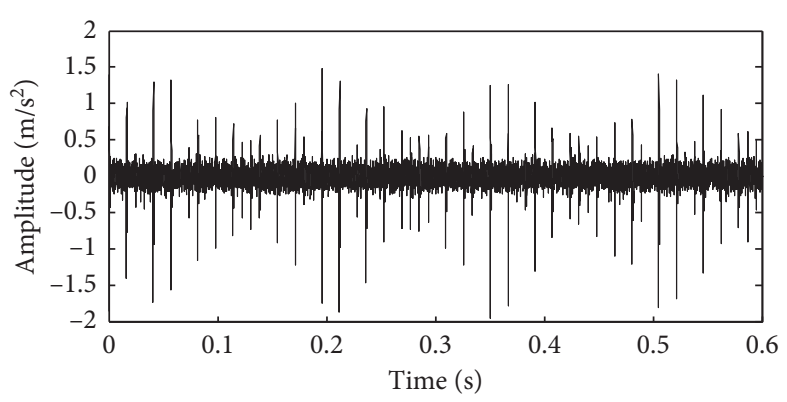

(a)

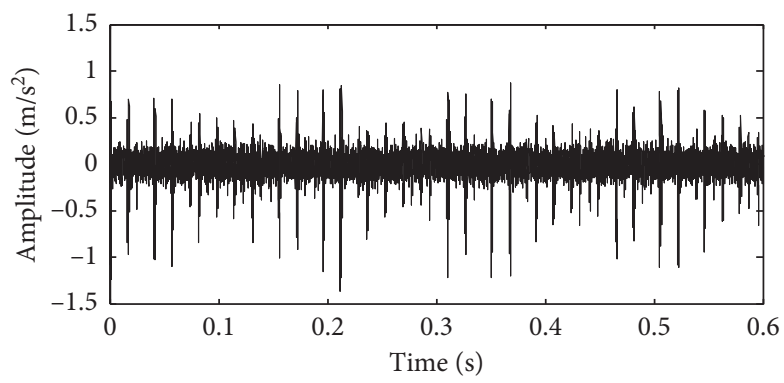

(c)

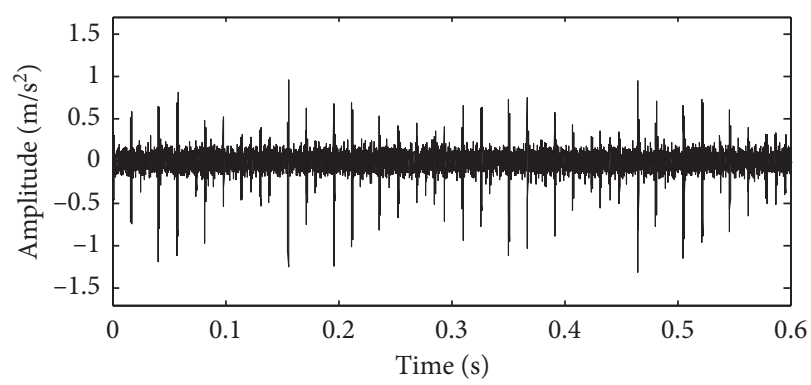

(b)

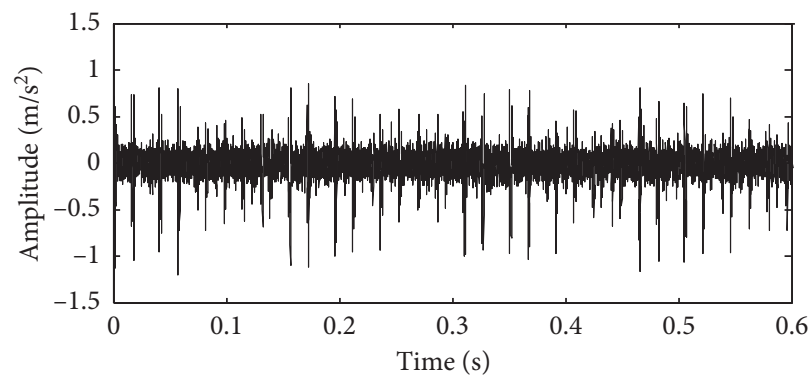

(d)

Figure 2: Simulation vibration signal of bearing inner race with different fault sizes. (a) Fault diameter: 0.5 mm. (b) Fault diameter: 2 mm. (c) Fault diameter: $3.5 \mathrm{~mm}$. (d) Fault diameter: $5 \mathrm{~mm}$.

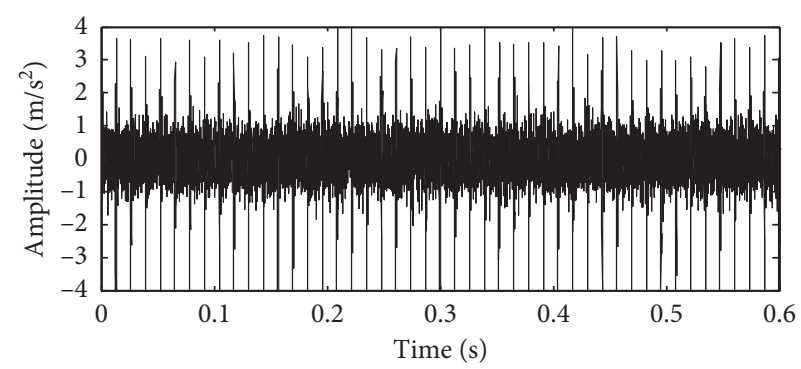

(a)

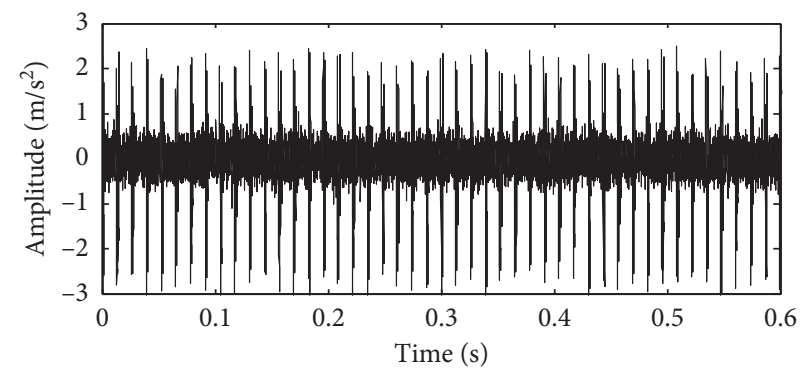

(c)

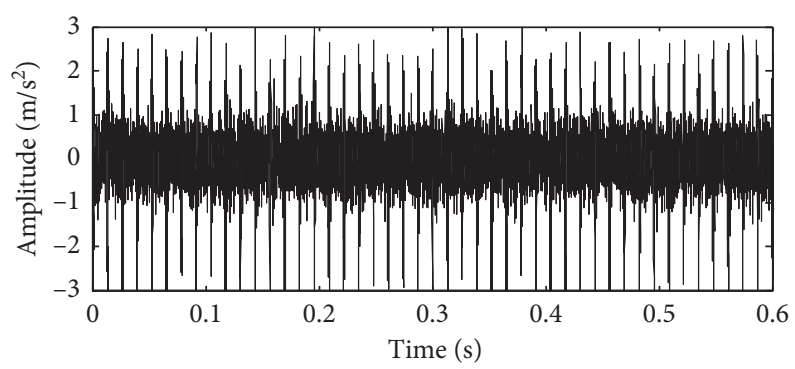

(b)

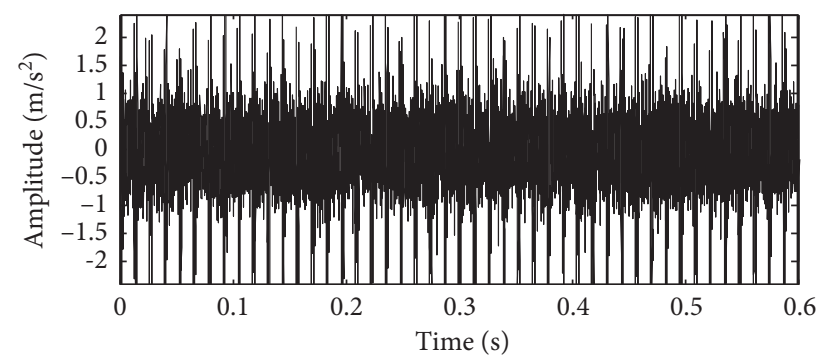

(d)

FIGURE 3: Simulation vibration signal of bearing outer race with different fault sizes. (a) Fault diameter: 0.5 mm. (b) Fault diameter: 2 mm. (c) Fault diameter: $3.5 \mathrm{~mm}$. (d) Fault diameter: $5 \mathrm{~mm}$.

of $N: N \geq 3600$, and $C_{n N}$ is normalized indicator of Lempel-Ziv.

3.2. The Protrugram Algorithm. Protrugram is an optimal frequency band selection method based on the kurtosis of the envelope amplitude of modulated signal. This method aims to select the optimal center frequency (CF) and bandwidth(BW).

The purpose of this method is to select the optimal center frequency and bandwidth. Firstly, FFT is performed on the measured time signal to obtain its spectrum. Second, the bandwidth is selected ([16] indicates that the bandwidth is usually 3 times of the fault characteristic frequency) and the 
iterative step length (usually $1 \mathrm{~Hz}, 100 \mathrm{~Hz}$, or $1000 \mathrm{~Hz}$ ). Subsequently, the interval [CF-BW/2, CF-BW/2] is selected on the FFT spectrum (positive part) during each iteration. Then, Hilbert envelope is used to obtain its envelope spectrum and calculate its kurtosis. Finally, the relationship between center frequency and kurtosis is plotted. The narrowest band signal with the maximum kurtosis is selected for subsequent analysis.

3.3. The Quantitative Trend Diagnosis of Bearing Fault Based on Protrugram and Lempel-Ziv. Firstly, the center frequency and bandwidth of the original signal is determined by the FFT transform. Then, the optimal analysis frequency band is selected and Lempel-Ziv complexity index of the frequency band is calculated. Finally, the relationship between Lempel-Ziv complexity and failure size is obtained.

The process of the method is as follows:

(1) FFT transform. The FFT transform is performed on the original fault signal of the bearing fault with different fault sizes.

(2) Initialization. Determine the bandwidth BW and the step size and the range of the center frequency $\mathrm{CF}$ $\left[\mathrm{BW} / 2, f_{\mathrm{s}} / 2-\mathrm{BW} / 2\right]$.

(3) Calculate the narrow-band envelope spectrum. The section of the spectrogram $\left[\mathrm{BW}-\mathrm{CF}_{\mathrm{k}}, \mathrm{BW}+\mathrm{CF}_{\mathrm{k}}\right]$ is subjected to IFFT transform and the envelope spectrum of the signal is calculated.

(4) Calculate the kurtosis value. Calculate the kurtosis value of the narrow-band envelope spectrum in the (3) step.

(5) Draw the graph. The correspondence between $\mathrm{CF}_{\mathrm{k}}$ as the abscissa and Kurtosis as the ordinate.

(6) Select the optimal frequency band. The frequency band with the largest kurtosis value is selected and the corresponding center frequency value $\mathrm{CF}_{\mathrm{o}}$ is returned to obtain the best analysis frequency band.

(7) Calculate the Lempel-Ziv value. Calculate the complexity index according to the method described in Section 3.1.

(8) Draw the relation graph between the Lempel-Ziv complexity value and fault size to realize the fault quantitative trend diagnosis.

The flowchart is shown in Figure 4.

\section{Applications to Bearing Fault Based on the Proposed Method}

4.1. Simulation Signal Analysis. From Figures 2 and 3, we can find that the time domain signals of the inner and outer race have obvious periodic impact. However, with the increase of fault severity, the fault signal of inner or outer race will not change significantly. Therefore, the quantitative fault diagnosis cannot be realized only by analyzing the time domain of fault signal.

For the outer race fault, the inherently attenuated vibration signal only related to the nature of the bearing itself,

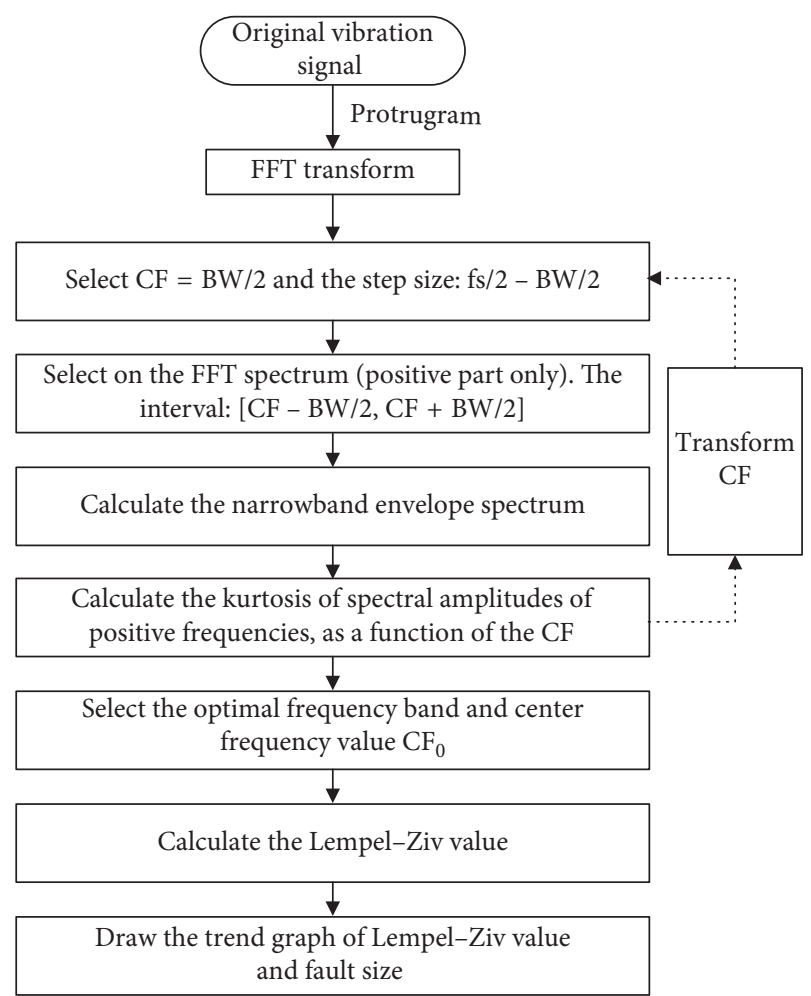

Figure 4: The flowchart of the proposed method.

and for the same size bearing, the inherent attenuation vibration signal can be considered unchanged; therefore, the pulse sequence $x_{\mathrm{f}}(t)$ is dominated by defects. As the severity of the fault increases, the width of each pulse in the impulse train increases. Therefore, when the impact frequency of the bearing defects remains unchanged, the duration of the large-amplitude vibration in the bearing signal will become longer and fuzzy before and after the superposition. With the increase of fault severity, the periodic impulse component of bearing signal will weaken, and the signal disturbance will increase [22]. This will lead to an increase in the randomness of the vibration signal $x(t)$ in the same time domain. With the increase of fault severity, the impact overlaps with each other, resulting in signal confusion and approaching random signals gradually, increasing the complexity. Therefore, with increase of the severity of the outer race fault, the Lempel-Ziv value should show an upward trend.

For the inner race fault, the fault position changes because the inner race rotates during bearing rotation. Therefore, the modulated signal caused by uneven load distribution will have a strong influence on the fault signal of the inner race. The pulse width increases as the fault severity increases. The combination of the pulse action and the nonuniform load distribution makes the periodicity of the signal envelope more obvious, that is, the modulation effect of the signal is enhanced. Therefore, with the increase of fault severity, the periodicity of signal envelope becomes more and more obvious, indicating that the signal order increases and the complexity decreases. In other words, with the increase of fault severity of inner race, Lempel-Ziv value should show a downward trend in theory. 
It can be seen from the above analysis that the fault signal components of bearing are relatively complex. Therefore, it is necessary to divide the analyzed signal into two parts, high frequency and low frequency, and calculate the complexity separately to ensure the accuracy of the calculation results. The overall complexity value of the bearing can represent the proportional sum of the high frequency complexity factor $C_{n N H}$ (original signal) and the low frequency (envelope of original signal) complexity coefficient $C_{n N L}$. The formula is

$$
C_{n}=W_{H} C_{n N H}+W_{L} C_{n N L} .
$$

Nikolaou [23] pointed out that due to the difference in the composition of the fault signal between the inner and outer race, the empirical values of the weight coefficients $W_{H}$ and $W_{H}$ are also different. For the inner race damage: $W_{L}=2 / 3, W_{H}=1 / 3$; for the outer ring damage: $W_{L}=1 / 2$, $W_{L}=1 / 2$.

Firstly, the outer race fault frequency is $76.73 \mathrm{~Hz}$, the bandwidth $\mathrm{BW}$ is determined to be $300 \mathrm{~Hz}$, the step length is $100 \mathrm{~Hz}$, and the range of the central frequency is $[150 \mathrm{~Hz}$, $7530 \mathrm{~Hz}$. The inner race fault frequency is $122.74 \mathrm{~Hz}$, the bandwidth BW is determined to be $400 \mathrm{~Hz}$, the step length is $100 \mathrm{~Hz}$, and the range of the central frequency is $[150 \mathrm{~Hz}$, $7530 \mathrm{~Hz}$ ]. according to Step 7 in Section 3.3.

The signals with different fault sizes are processed to obtain narrow-band signals and the Lempel-Ziv value of narrow-band signals is calculated. Figure 5 shows the trend of different Lempel-Ziv values for inner and outer race failures.

From Figure 5, when the fault size increases, the Lempel-Ziv complexity index of the outer race fault signal shows an upward trend, and the Lempel-Ziv complexity index of the inner race fault signal shows a downward trend [21]. This indicates that the Lempel-Ziv value of the signal produced by the different bearing inner and outer race faults can be used to realize the quantitative fault diagnosis of the bearing.

But in the process of dealing the measured signal because of the useless vibration interference, it cannot get the same result as the simulation signal only by using Lempel-Ziv. So, the quantitative trend diagnosis method of rolling bearing fault based on Protrugram and Lempel-Ziv is proposed.

4.2. Experimental Verification. The experimental data come from the bearing test bench of the laboratory, and we cited this original experimental signal on pages 7 to 8 of the paper [21]. The experimental bench's components (shown in Figure 6) are described in Table 1. Table 2 shows the parameters of the bearing. Four sizes of rolling bearing fault are obtained by electrosparking manually; they are $0.5 \mathrm{~mm}$, $2 \mathrm{~mm}, 3.5 \mathrm{~mm}$ and $5 \mathrm{~mm}$, and experimental data of the inner and outer race fault under the condition of each fault size are obtained through acceleration sensor. Setting sampling frequency as $12800 \mathrm{~Hz}$, the sampling number $N$ is 8192 . The investigated faulty bearing with fault size of $0.5 \mathrm{~mm}$ is shown in Figure 7.

4.2.1. Analysis of Inner Race Fault Data. Firstly, the inner race fault frequency is $123.738 \mathrm{~Hz}$, the bandwidth $\mathrm{BW}$ is

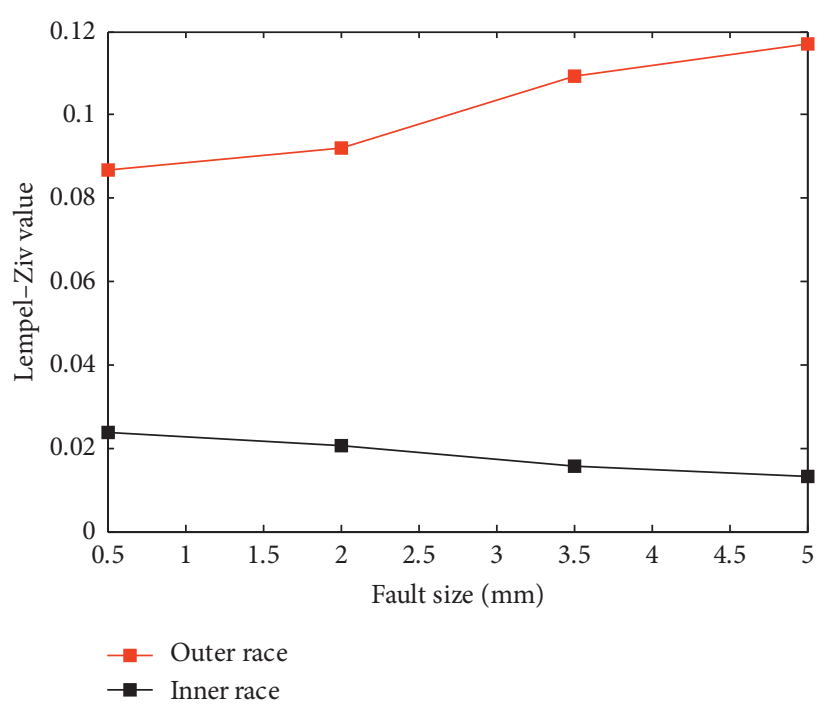

Figure 5: The Lempel-Ziv trend chart of the original signal.

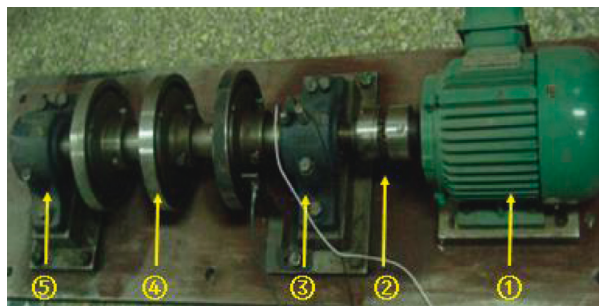

Figure 6: The experimental bench system.

TABLE 1: The test bench components.

\begin{tabular}{lc}
\hline (1) & Three-phase asynchronous motor \\
(2) & Flexible shaft coupling \\
(3) & The normal bearing \\
(4) & Bearing rotor \\
(5) & The bearings with different fault size \\
\hline
\end{tabular}

TABLE 2: The geometric parameters of the tested bearing.

\begin{tabular}{lc}
\hline Outer diameter of bearings $D_{0}$ & $80 \mathrm{~mm}$ \\
Inner diameter of bearings $d_{0}$ & $35 \mathrm{~mm}$ \\
Contact angle $\alpha$ & $0^{\circ}$ \\
Number of rolling elements $Z$ & 8 \\
Motor speed R & $1497 \mathrm{r} / \mathrm{min}$
\end{tabular}

determined to be $400 \mathrm{~Hz}$, the step length is $100 \mathrm{~Hz}$, and the range of the central frequency is $[200 \mathrm{~Hz}, 6200 \mathrm{~Hz}]$. The signals with different fault sizes are processed to obtain narrow-band signals, and the Lempel-Ziv value of narrowband signals is calculated. Then, the trend graph of Lempel-Ziv complexity indicator is drawn, as shown in Figure 8 .

From Figure 8, it can be seen that the trend graph of Lempel-Ziv complexity of narrow-band signals shows the complete downward trend with better effect. On the other hand, the Lempel-Ziv complexity index of narrow-band 


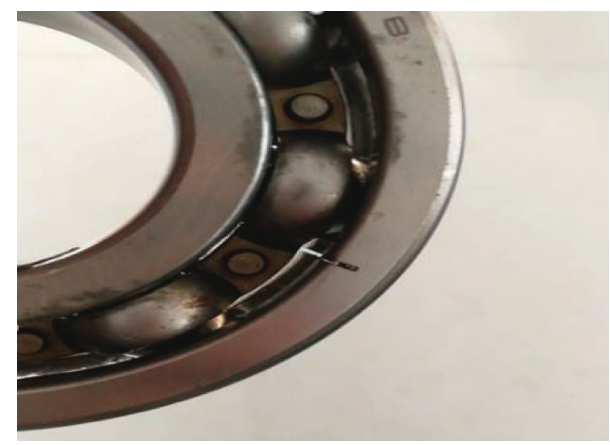

Figure 7: The fault diameter $0.5 \mathrm{~mm}$ outer race defect of the test rolling bearing.

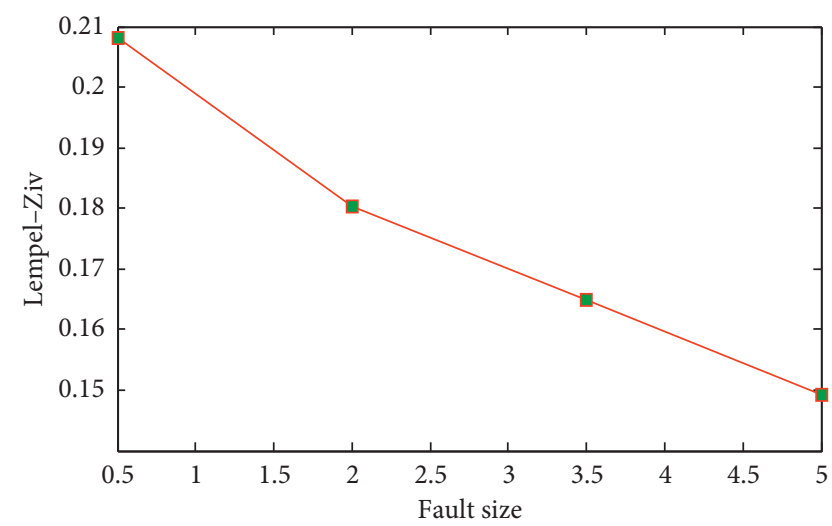

Figure 8: Lempel-Ziv trend graph of inner race defect.

signals has a very large trend slope, which has a significant impact on the quantitative trend diagnosis of rolling bearings.

Therefore, this method is more effective in diagnosing the trend of bearing failure. In addition, it can be inferred from the reverse that if the complexity of a series of fault signals decreases after noise reduction, the set of signals is the roller bearing signals with inner race, so as to realize fault recognition and quantitative trend diagnosis.

4.2.2. Analysis of Outer Race Fault Data. Firstly, the outer race fault frequency is $78.7282 \mathrm{~Hz}$, the bandwidth $\mathrm{BW}$ is determined to be $300 \mathrm{~Hz}$, the step length is $100 \mathrm{~Hz}$, the value range of the central frequency is $[150 \mathrm{~Hz}, 6250 \mathrm{~Hz}]$, and 8192 points are intercepted. The signals with different fault sizes are processed to obtain narrow-band signals, and the Lempel-Ziv value of narrow-band signals is calculated. Then, the trend diagram of Lempel-Ziv complexity indicator is drawn, as shown in Figure 9.

From Figure 9, it can be seen that the trend graph of Lempel-Ziv complexity of narrow-band signals shows the complete upward trend with better effect. On the other hand, the Lempel-Ziv complexity index of narrow-band signals has a very large trend slope, which has a significant impact on the quantitative trend diagnosis of rolling bearings.

Therefore, this method is more effective in diagnosing the trend of bearing failure. In addition, it can be inferred

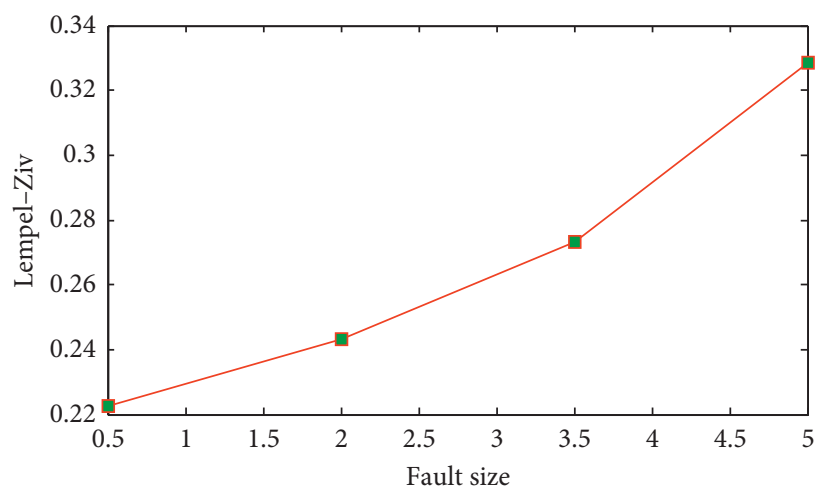

Figure 9: The Lempel-Ziv trend diagram of outer race defect.

from the reverse that if the complexity of a series of fault signals increases after noise reduction, the set of signals is roller bearing signals with outer race, so as to realize fault recognition and quantitative trend diagnosis.

\section{Conclusion}

A quantitative trend diagnosis method based on Protrugram and Lempel-Ziv is proposed for rolling bearing fault. This paper introduces the Protrugram algorithm to extract the rolling bearing fault feature and select the optimal analysis frequency band as the narrow-band signal, then calculates the Lempel-Ziv complexity index of narrow-band signal and gives the relationship between fault size and Lempel-Ziv value to realize the quantitative trend diagnosis of rolling bearing. The Lempel-Ziv indicator can measure the complexity of the signal. The experimental analysis proves the superiority of this method in the quantitative trends diagnosis of bearing fault.

\section{Data Availability}

The experimental data [.txt] used to support the findings of this study were supplied by the research group of Key Laboratory of Advanced Manufacturing Technology under license and so we still cannot put it into the system, and the requests for access to these data should be made to the corresponding author.

\section{Conflicts of Interest}

The authors declare that they have no conflicts of interest.

\section{Acknowledgments}

This work was supported by the National Natural Science Foundation of China (Grant no.51575007).

\section{References}

[1] Y. Hu, X. Tu, F. Li et al., "An adaptive and tacholess order analysis method based on enhanced empirical wavelet transform for fault detection of bearings with varying speeds," Journal of Sound and Vibration, vol. 409, pp. 241-255, 2017. 
[2] Z. Feng, M. J. Zuo, R. Hao et al., "Ensemble empirical mode decomposition-based teager energy spectrum for bearing fault diagnosis," Journal of Vibration and Acoustics-Transactions of the ASME, vol. 135, no. 3, article 031013, 2013.

[3] L. Song, H. Wang, and P. Chen, "Step-by-step fuzzy diagnosis method for equipment based on symptom extraction and trivalent logic fuzzy diagnosis theory," IEEE Transactions on Fuzzy Systems, vol. 1, pp. 1-13, 2018.

[4] L. Song, H. Wang, and P. Chen, "Vibration-based intelligent fault diagnosis for roller bearings in low-speed rotating machinery," IEEE Transactions on Instrumentation \& Measurement, vol. 67, no. 8, pp. 1887-1899, 2018.

[5] L. Cui, Y. Zhang, F. Zhang et al., "Vibration response mechanism of faulty outer race rolling element bearings for quantitative analysis," Journal of Sound \& Vibration, vol. 364, no. 3, pp. 67-76, 2016.

[6] K. Jiang, G. Xu, L. Liang et al., "A quantitative diagnosis method for rolling element bearing using signal complexity and morphology filtering," Journal of Vibroengineering, vol. 14, no. 4, pp. 1862-1875, 2012.

[7] X. Zhang, Y. Liang, J. Zhou et al., "A novel bearing fault diagnosis model integrated permutation entropy, ensemble empirical mode decomposition and optimized SVM," Measurement, vol. 69, pp. 164-179, 2015.

[8] L. Cui, N. Wu, C. Ma et al., "Quantitative fault analysis of roller bearings based on a novel matching pursuit method with a new step-impulse dictionary," Mechanical Systems \& Signal Processing, vol. 68-69, pp. 34-43, 2016.

[9] W. Gao, C. Li, and Z. Zhang, "Complexity analysis of vibration signals for gearbox," Journal of Electronic Measurement and Instrument, vol. 16, no. 2, pp. 1-4, 2002.

[10] H. Hong and M. Liang, "Fault severity assessment for rolling element bearings using the Lempel-Ziv complexity and continuous wavelet transform," Journal of Sound \& Vibration, vol. 320, no. 1, pp. 452-468, 2009.

[11] D. Dou and Y. Zhao, "Study on damage recognition of rolling bearing based on EMD and Lempel-Ziv index," Journal of Vibration and Shock, vol. 29, no. 3, pp. 5-8, 2010.

[12] C. Zhang and J. Chen, "Research on severity of damage of rolling bearing based on LMD and Lempel-Ziv index," $V i$ bration and Shock, vol. 31, no. 16, pp. 77-82, 2012.

[13] Y. Hu, F. Li, H. Li et al., "An enhanced empirical wavelet transform for noisy and non-stationary signal processing," Digital Signal Processing, vol. 60, pp. 220-229, 2017.

[14] Y. Liu, Z. Dai, and S. Lu, "Enhanced bearing fault detection using step-varying vibrational resonance based on duffing oscillator nonlinear system," Shock and Vibration, vol. 2017, Article ID 5716296, 14 pages, 2017.

[15] K. Li, L. Su, J. Wu et al., "A rolling bearing fault diagnosis method based on variational mode decomposition and an improved kernel extreme learning machine," Applied Sciences, vol. 7, no. 10, p. 1004, 2017.

[16] T. Barszcz and A. Jabłoński, "A novel method for the optimal band selection for vibration signal demodulation and comparison with the Kurtogram," Mechanical Systems \& Signal Processing, vol. 25, no. 1, pp. 431-451, 2011.

[17] Y. Miao, M. Zhao, and J. Lin, "Improvement of kurtosisguided-grams via Gini index for bearing fault feature identification," Measurement Science and Technology, vol. 28, no. 12, 2017.

[18] L. Cui, J. Huang, and F. Zhang, "Quantitative and localization diagnosis of a defective ball bearing based on verticalhorizontal synchronization signal analysis," IEEE
Transactions on Industrial Electronics, vol. 64, no. 11, pp. 8695-8706, 2017.

[19] L. Cui, J. Wang, and S. Lee, "Matching pursuit of an adaptive impulse dictionary for bearing fault diagnosis," Journal of Sound and Vibration, vol. 333, no. 10, pp. 2840-2862, 2014.

[20] R. Yan and R. X. Gao, "Complexity as a measure for machine health evaluation," IEEE Transactions on Instrumentation and Measurement, vol. 53, no. 4, pp. 1327-1334, 2004.

[21] L. Cui, B. Li, J. Ma et al., "Quantitative trend fault diagnosis of a rolling bearing based on Sparsogram and Lempel-Ziv," Measurement, vol. 128, pp. 410-418, 2018.

[22] L. Cui, X. Gong, J. Zhang et al., "Double-dictionary matching pursuit for fault extent evaluation of rolling bearing based on the Lempel-Ziv complexity," Journal of Sound and Vibration, vol. 385, pp. 372-388, 2016.

[23] N. G. Nikolaou, "Rolling element bearing fault diagnosis using wavelet packets," NDT\&E International, vol. 35, no. 3, pp. 197-205, 2002. 


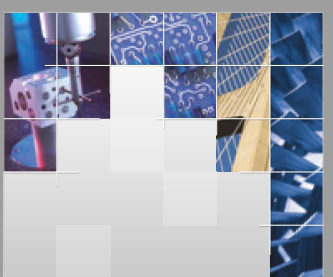

\section{Enfincering}
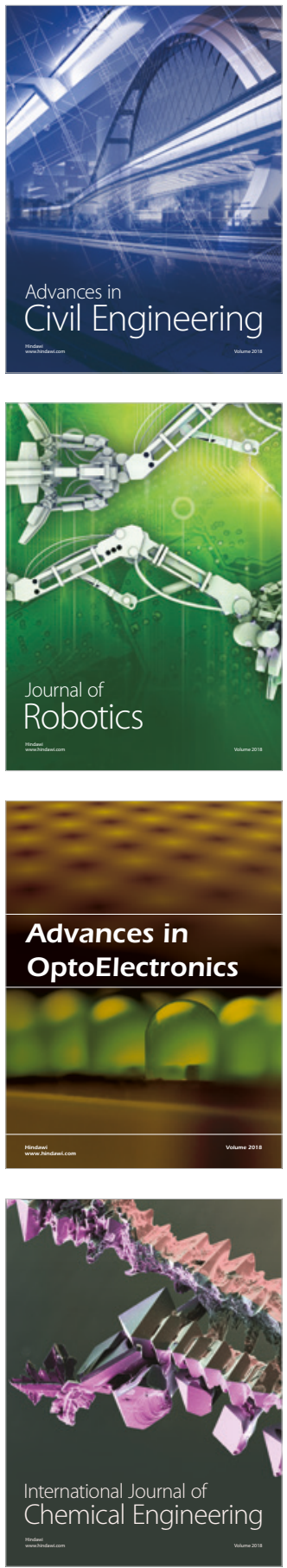

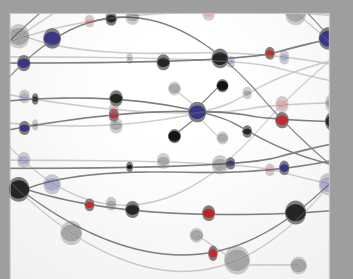

\section{Rotating \\ Machinery}

The Scientific World Journal

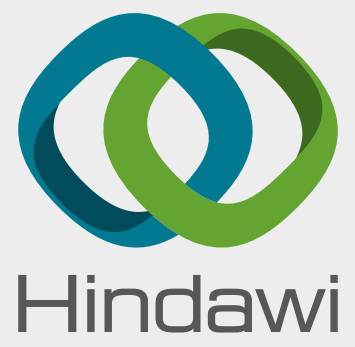

Submit your manuscripts at

www.hindawi.com
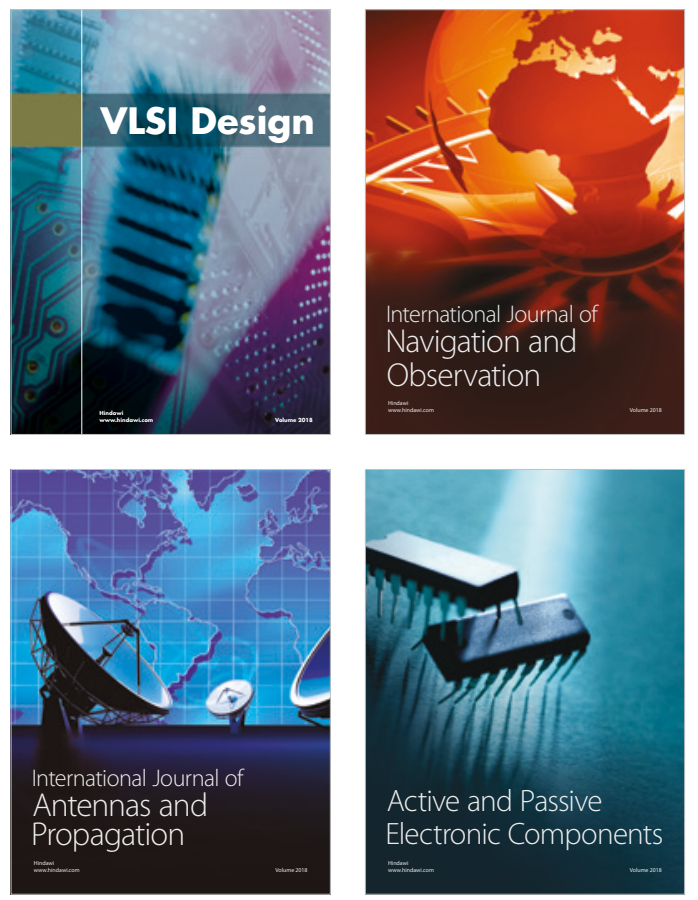
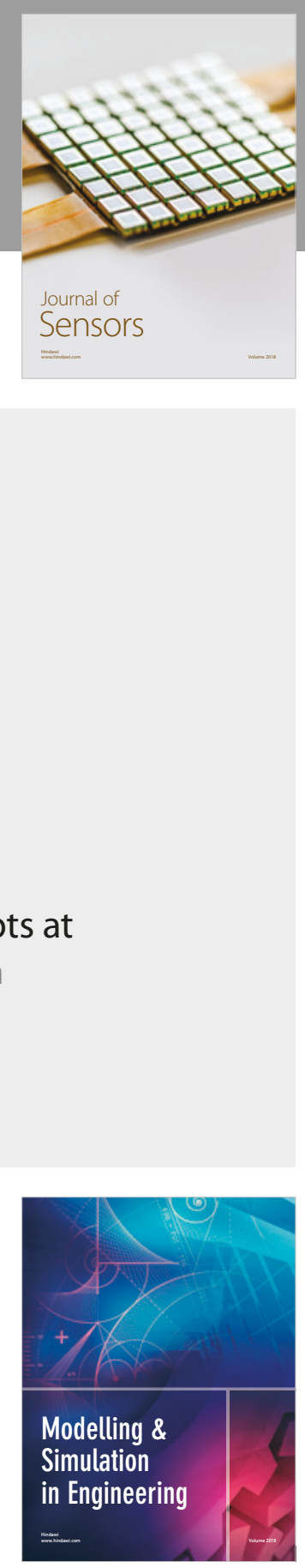

\section{Advances \\ Multimedia}
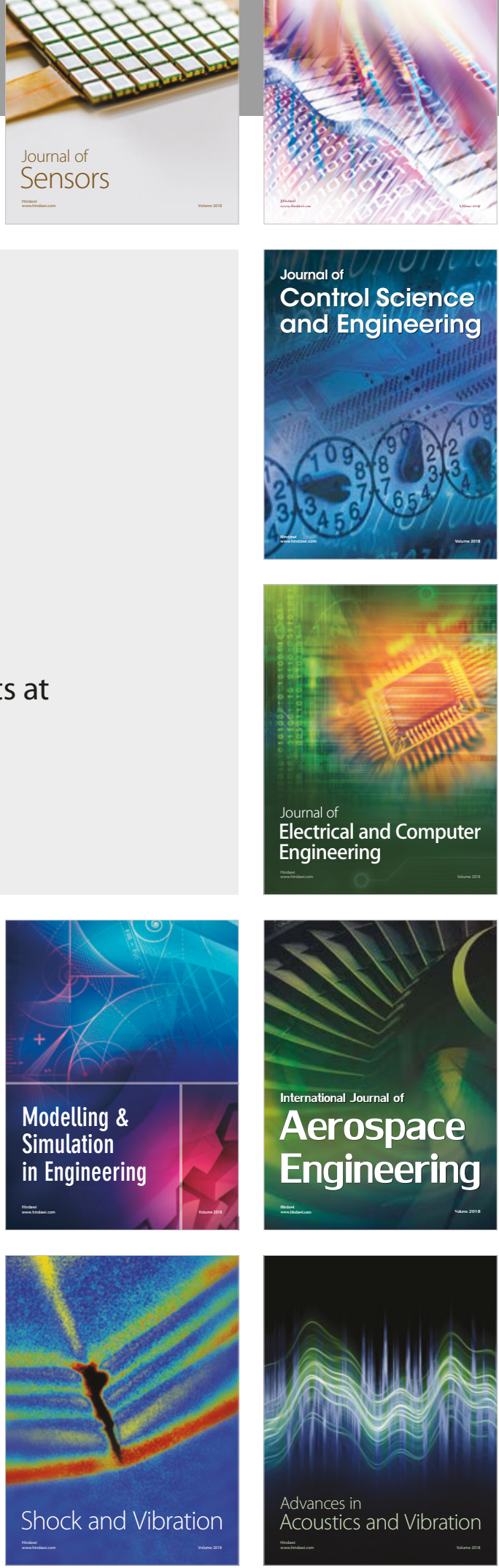